\title{
Hearing impairment and neurological dysfunction associated with a mutation in the mitochondrial tRNA ${ }^{\operatorname{Ser}(\mathrm{UCN})}$ gene
}

\author{
K ristien Verhoeven ${ }^{1}$, R obbert J H Ensink ${ }^{2}$, Valeria Tiranti ${ }^{3}$, Patrick LM H uygen ${ }^{2}$, \\ $D$ avid F J ohnson ${ }^{4}$, I sabelle Schatteman ${ }^{1}$, L ut Van L aer ${ }^{1}$, M argriet Verstreken ${ }^{1}$, \\ Paul Van de Heyning ${ }^{5}$, Nathan Fischel-G hodsian ${ }^{4}, \mathrm{M}$ assimo Zeviani ${ }^{3}$, \\ Cor WRJ Cremers ${ }^{2}$, Patrick J Willems ${ }^{1}$ and Guy Van Camp ${ }^{1}$ \\ ${ }^{1}$ D epartment of M edical G enetics, U niversity of A ntwerp (UIA), B elgium \\ ${ }^{2} \mathrm{D}$ epartment of $\mathrm{O}$ tolaryngology, U niversity $\mathrm{H}$ ospital $\mathrm{Nijmegen,} \mathrm{The} \mathrm{N}$ etherlands \\ ${ }^{3}$ D ivision of B iochemistry and G enetics, National N eurological I nstitute 'C arlo B esta', M ilan, I taly \\ ${ }^{4} \mathrm{M}$ edical $\mathrm{G}$ enetics B irth D efects Center, Departments of M edicine and Pediatrics, Cedars-Sinai M edical Center and \\ UCL A School of Medicine, L os A ngeles, CA, USA \\ ${ }^{5}$ E NT D epartment, U niversity Hospital of A ntwerp, B elgium
}

\begin{abstract}
We studied a large $D$ utch family with maternally inherited, progressive, sensorineural hearing loss in 27 patients. 0 nly in a single family member was the hearing loss accompanied by neurological symptoms including ataxia and dysarthria. DNA analysis of the mitochondrial genome revealed the insertion of a $C$ at nucleotide position 7472 in the tR NA ${ }^{\text {Ser(UCN) }}$ gene (7472insC mutation). We determined the percentage of mutant D NA (heteroplasmy) in blood from all family members, and found no correlation between hearing loss and leucocyte heteroplasmy. The 7472insC mutation was previously identified in a smaller family from Sicily with sensorineural hearing loss in 9 family members, six of them also presenting neurologically with ataxia and myoclonus. The presence of the 7472insC mutation in two different pedigrees strongly supports its pathogenicity. $\mathrm{H}$ owever, the interfamilial difference in penetrance of the neurologic abnormalities is most likely to be strongly influenced by secondary factors different from the 7472insC mutation, as heteroplasmy or age of the patients were similar in both families. This mutation should therefore be analysed in families with maternally inherited hearing loss, irrespective of whether the hearing loss is non-syndromic or accompanied by neurologic abnormalities.
\end{abstract}

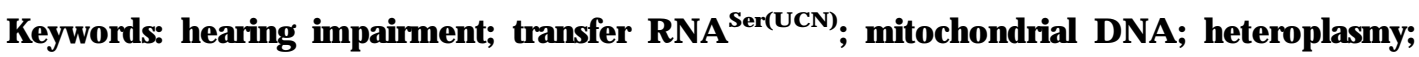
ototoxicity

Correspondence: Guy Van Camp, Department of Medical G enetics, U niversity of A ntwerp (UIA ), U niversiteitsplein 1, 2610 Wilrijk, Belgium. Tel: 00323 8202570; Fax: 00323 8202566; E-mail: gvcamp@uia.ua.ac.be

R eceived 30 January 1998; revised 15 J une 1998; accepted 19 J une 1998 


\section{Introduction}

Several human tissues and organs like the brain, retina, muscle, kidney and endocrine glands are highly dependent on the energy produced by mitochondrial oxidation. As a consequence these tissues are more vulnerable to mitochondrial defects. ${ }^{1,2}$ In almost all mitochondrial diseases identified so far one or more of these tissues or organs are involved. In many of these, hearing loss is an accompanying symptom. This is probably caused by a high reliance of the inner ear on mitochondrial energy production. ${ }^{3}$

Until now only three different mutations in the mitochondrial genome have been reported to cause hearing loss as a sole or predominant symptom. The first mutation, an $A$ to $G$ substitution at position 1555 in the $12 S$ rRNA gene $(1555 \mathrm{~A} \rightarrow \mathrm{G})$, was reported in a large I sraeli-A rab family with maternally inherited, non-syndromic hearing loss. ${ }^{3}$ This mutation was also found in many other pedigrees with non-syndromic deafness, ${ }^{4-6}$ and in patients and families with aminoglycoside-induced ototoxic hearing impairment., ${ }^{4,7}$ A pparently this mutation makes individuals susceptible to hearing impairment after treatment with aminoglycosides at concentrations that normally do not affect hearing. Even without exposure to aminoglycosides many patients with this mutation develop hearing impairment, but it is currently not known which other factors are involved in the etiology.

A second mutation of the mitochondrial genome was described in a Scottish family with maternally-inherited, bilateral, sensorineural hearing loss. The mutation substituted a $G$ for an $A$ at position $7445(7445 \mathrm{~A} \rightarrow \mathrm{G})$ of the mtD NA ${ }^{10}$ and has subsequently been observed in a second pedigree from $\mathrm{N}$ ew Z ealand. ${ }^{11}$

The insertion of $\mathrm{a} C$ at nucleotide position 7472 in the tRNA ${ }^{\text {Ser(UCN) }}$ gene (7472insC) has been reported in a Sicilian family with maternally-inherited hearing loss as a predominant feature. ${ }^{12}$ In six out of nine patients the hearing loss was accompanied by ataxia, dysarthria and focal myoclonus.

In the present study we report a second family with the 7472insC mutation. A lthough neurological abnormalities were found in only a single patient from this large family, no significant difference in heteroplasmy was found between this family and the first family from Sicily, in which seven patients have neurological problems. These results indicate that the strongly reduced penetrance of neurological symptoms in this family is most likely caused by other factors besides the 7472insC mutation.

\section{Patients and Methods}

Audiometric Studies

Sixty-four family members including 10 spouses participated in this study (Figure 1). A general otological examination and pure tone audiometry with air and bone conduction was performed. Pure tone average hearing thresholds at $0.25,0.5$, $1,2,4$ and $8 \mathrm{kHz}$ were determined. Patients with a hearing threshold below the 95th percentile of an age and sex dependent audiometric curve (ISO) were considered to be hearing impaired. Family members with a hearing threshold better than $20 \mathrm{~dB}$ or above the 50th percentile were considered to have normal hearing.

Twenty-seven family members were found to be hearing impaired. Their hearing loss was accompanied by tinnitus in the majority of patients, and by dizziness in about one third of patients. Two family members (I:1 and II:16) showed a documented acute drop in their hearing levels after treatment with streptomycin for tuberculous spondylitis and pneumonia, respectively. A third family member from the maternal line (who did not participate in this study) had heteroanamnestically an acute loss of hearing after 2 weeks of treatment with streptomycin for tuberculous pneumonia. 0 ne patient with hearing loss (11:30) had a history of noise exposure and was therefore given an unknown affection status.

In all patients, hearing loss was sensorineural and progressive, mainly affecting the high frequencies in the initial stage and gradually affecting lower frequencies with increasing age. Hearing loss was first noted in the patients between 12 and 45 years of age. Nearly all family members from the maternal line older than 30 were affected, except for four family members, between 38 and 47 years old $(I I-3,1 I-6, \mid I-24$, II-26).

\section{Clinical Examination}

All family members were interviewed about their clinical history, and the presence of possible neurological complaints was investigated by questionnaire. Nine different family members of the maternal line were selected for a neurological examination. E ight family members ranging in age from 35 to 55 had no neurological abnormalities, but most of them showed a mild general neurological picture which can best be described as clumsiness. Only the proband (patient 1-5) had truncal and limb ataxia, dysarthria and motor and sensory polyneuropathy in the limbs. MRI of the proband's brain showed atrophy of the cerebellar vermis and multiple subcortical white matter lesions. Two deceased brothers of the proband had suffered from a neurological picture similar to that of the proband. However, no clinical data could be recovered from these deceased patients. O phthalmological examination of four patients was normal with no evidence of tapetoretinal degeneration. Three patients $(\mid-4,1-5,1-13)$ reported diabetes mellitus type II.

\section{MtDNA Mutation Analysis}

Blood samples were taken after informed consent. Total DNA was extracted from blood samples by standard techniques. To detect large mitochondrial rearrangements, Southern blot analysis was performed. The presence of the $7445 \mathrm{~A} \rightarrow \mathrm{G}$ and $1555 \mathrm{~A} \rightarrow \mathrm{G}$ mutations associated with hearing loss, was investigated. ${ }^{3,10} \mathrm{~A}$ nalysis of the 7472 ins $C$ mutation in 


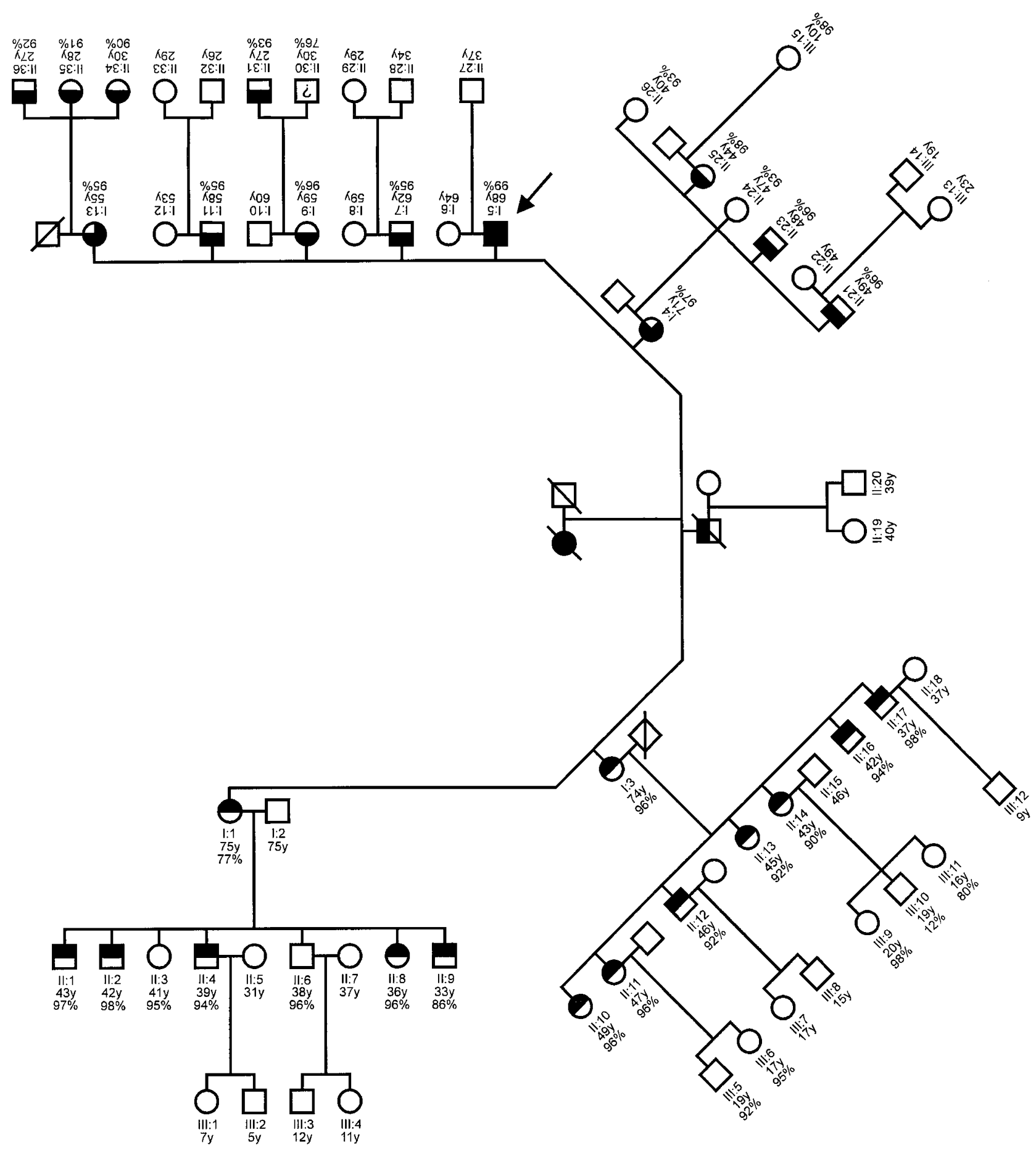

Figure 1 Pedigree of the D utch family members who participated in this study. O nly the family members from whom a D NA sample was available are numbered. In all family members of the maternal line the presence of the 7472insC mutation was demonstrated. Patients with hearing loss only are indicated by a half-filled symbol $(\theta, \theta)$. Patients with hearing loss and diabetes mellitus type II are indicated by a three-quarter filled symbol $(\boldsymbol{C}, \mathbf{E})$. The patient with hearing loss, diabetes mellitus and neurological symptoms is indicated by a solid symbol ( $\square$ ). U naffected family members are indicated with open symbols $(\square, 0)$. Individual II-30 had hearing loss probably caused by noise exposure, and was therefore given an unknown affection status (?). The ages of the living family members and the percentage of mutant mitochondrial DNA are indicated below the symbols. 
the tRNA ${ }^{\operatorname{Ser}(U C N)}$ gene was done by modified PCR amplification followed by digestion with $\mathrm{Xcml}$, as previously described. ${ }^{12}$

In order to determine the percentage of mutant mtD NA, solid phase mini sequencing (SPM S) analysis was carried out as described previously. ${ }^{12}$ DNA sequencing of the PCR fragment containing the 7472insC mutation was performed on a Perkin-Elmer automated DNA sequencer using dyeprimer cycle sequencing kits (PE).

\section{Statistical Analysis}

We wished to find out whether there was a significant correlation between the percentage of mutant mtD NA and the hearing loss. B ecause the percentage of mutant mtD NA was clearly not normally distributed, we used nonparametric statistics. Spearman's rank correlation test was used to evaluate the correlation and the $M$ ann-W hitney test was used for comparing differences in heteroplasmy between groups of patients with different symptoms and signs. The level of significance used was $P=0.05$ in all tests.

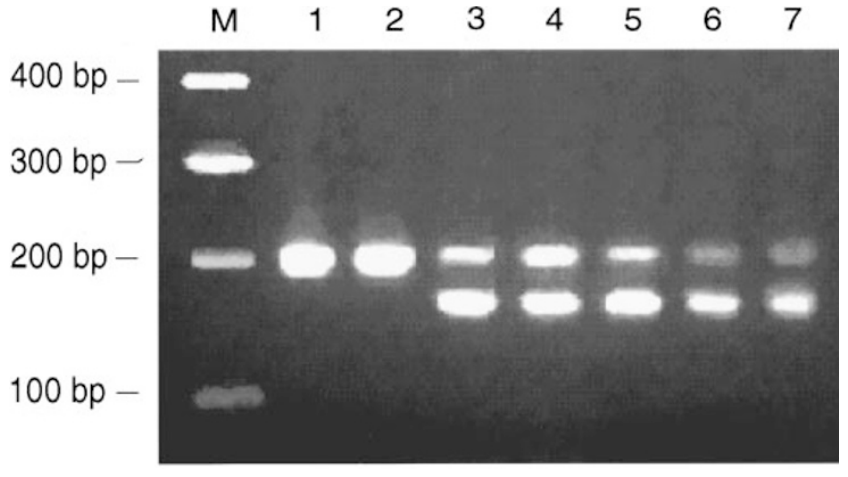

Figure 2 PCR amplification and $X \mathrm{cml}$ digestion of a mitochondrial DNA fragment (position 7432-7640). In patients II-9, I-1, I-3, II-12 and I-13 with the 7472insC mutation (lanes 3 to 7), the $208 \mathrm{bp} \mathrm{PCR}$ product is cut into two fragments of $168 \mathrm{bp}$ and $40 \mathrm{bp}$. The $40 \mathrm{bp}$ fragment is not visible on the gel. $\mathrm{D}$ ue to heteroplasmy, the undigested fragment remains visible. $L$ anes 1 and 2 show controls in which the $208 \mathrm{bp}$ product remains undigested. The left lane (M) contains a $100 \mathrm{bp}$ ladder as size standard.

bers of the maternal lineage, including those without hearing loss, were tested by PCR and restriction digest with $\mathrm{X} \mathrm{cml}$. They all showed the 7472insC mutation (Figure 1). O ne hundred unrelated normal controls were also tested, but we did not detect the 7472insC mutation.

In most family members with the 7472insC mutation the normal band remains visible, suggesting heteroplasmy for the mutation. Therefore, the percentages of wild-type and mutant lymphocyte mtDNA were analysed by SPMS. The percentage of mutant mtDNA observed in each family member of the maternal line is shown in Figure 1. A lthough the heteroplasmy ranges from 12 to $99 \%$ mutant mtD NA, the majority of family members showed values between 90 and $99 \%$. In the proband (I-5) the heteroplasmy was determined in lymphocytes as well as in muscle. No difference between percentage of mutant lymphocyte and muscle mtD NA was found.

\section{Statistical Analysis}

We statistically tested a possible correlation between the degree of hearing impairment at the low frequencies $0.25,0.5,1 \mathrm{kHz}$ and at the high frequencies 2 , 4 and $8 \mathrm{kHz}$ and the percentage of heteroplasmy. The Spearman's rank correlation test was not significant ( $n=38, r=0.26$ and 0.12 ) for a correlation between the degree of hearing impairment and heteroplasmy 


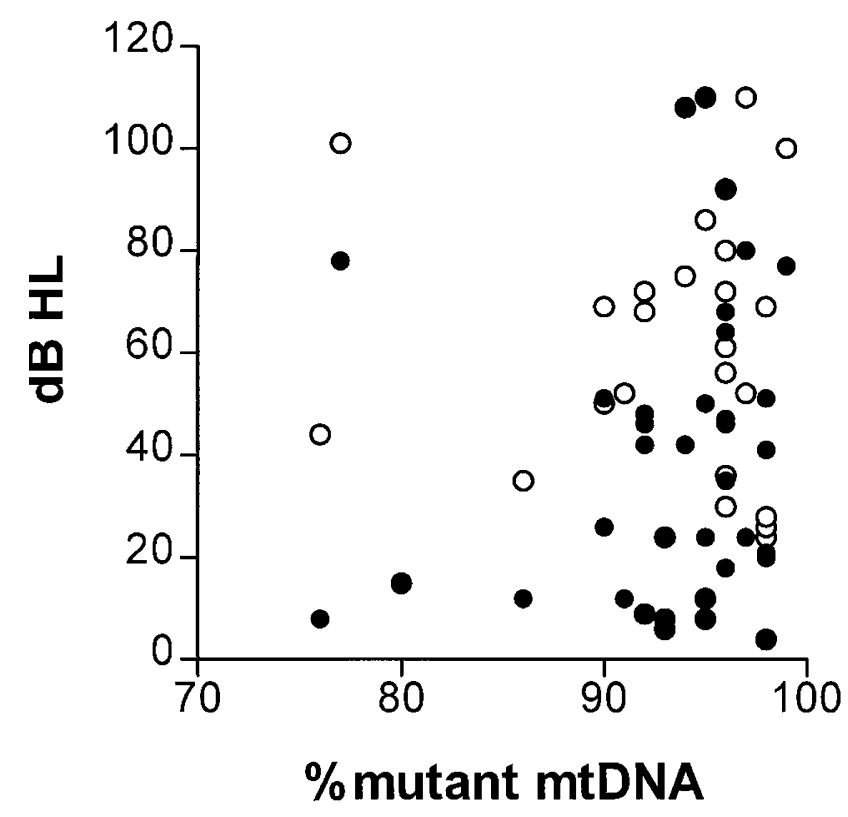

Figure $3 \mathrm{H}$ earing threshold $(\mathrm{dB} H \mathrm{H})$ plotted against percentage of mutant $\mathrm{mtD} N \mathrm{~A}$ in 37 members of the family. A verage hearing thresholds for the frequencies $0.25,0.5$ and $1 \mathrm{kHz}$ are indicated by a full circle (0); average hearing thresholds at frequencies 2, 4 and $8 \mathrm{kHz}$ are indicated by an open symbol (○). Patient III-10 (12\%, $10 \mathrm{~dB}$ low, $10 \mathrm{~dB}$ high) is not included in the figure for clarity.

(Figure 3). We also failed to find a significant correlation between age and heteroplasmy (data not shown).

\section{Analysis of Patients with Aminoglycoside Ototoxicity}

A s three family members with the 7472insC mutation developed hearing loss after the use of aminoglycosides, we investigated the possibility that this mutation causes susceptibility to aminoglycoside ototoxicity. We analysed the presence of this mutation in 52 patients who suffered from acute hearing loss after the use of aminoglycosides. ${ }^{13}$ Forty-three of these patients were unrelated. In seven of them the $1555 \mathrm{~A} \rightarrow \mathrm{G}$ mutation was present, but the 7472insC mutation was not detected in any of the patients.

\section{Discussion}

We found the previously reported mitochondrial mutation 7472insC in a Dutch family with maternally inherited sensorineural, bilateral hearing loss. The fact that the mutation is present in two independent families with hearing loss as a predominant symptom ${ }^{12}$ is strong evidence for the pathogenic role of the
7472insC mutation. A dditional support for the diseasecausing nature of this mutation includes:

i) the segregation pattern of the hearing loss in the large D utch family is clearly mitochondrial,

ii) the mutation was present in all members of the maternal lineage of the Dutch and the Italian family,

iii) the mutation was not present in any of the 100 D utch control persons in this study, nor in any of the 381 Italian control persons in a previous study, ${ }^{12}$

iv) the mutation changes the conformation of the conserved T $\Psi C$ loop in the secondary structure of the tRNA ${ }^{\text {Ser(UCN) }}$ gene,

v) using homoplasmic cybrids it was previously shown that the mutation reduces oxidative phosphorylation, ${ }^{12}$ and

vi) the mutation in both families is heteroplasmic, which is considered to be an indication of the pathogenicity of a mutation. ${ }^{14}$

This mutation was previously reported in a Sicilian family. Of the thirteen members of the Sicilian family with the mutation, four were unaffected, two had only hearing loss, and seven had neurological symptoms, four being severely neurologically affected. ${ }^{12}$ In the Dutch family, 27 of the 38 family members with the mutation had hearing loss as an isolated symptom. A II family members above the age of 30 with the mutation had hearing loss, except for four persons. The fact that these four family members have normal hearing might be due to a highly variable age of onset or to reduced penetrance of the hearing impairment. In the Dutch family only the index patient (I-5) has ataxia of both limbs and dysartheria in addition to hearing loss. So the Dutch family has a lower penetrance of neurological symptoms than the Sicilian family.

We analysed the percentages of heteroplasmy in the previously described Sicilian family and the Dutch family in relation to the presence or absence of hearing impairment or neurological impairment in patients aged 25 years and over. There was a significant difference in the percentage of heteroplasmy between the family members with and without hearing/neurological impairment in the Sicilian family ( $M$ ann-Whitney test, $P<0.05)$. In the Sicilian family, a threshold of approximately $95 \%$ was suggested for the manifestation of all 
neurological symptoms in addition to hearing loss. ${ }^{12}$ H owever, in the D utch family, 20 family members with $95 \%$ heteroplasmy or more, had no additional neurological symptoms. Only the proband, who had the highest percentage of heteroplasmy (99\%), had a clear neurological picture.

However, it is very possible that a higher threshold ( $99 \%$ ) for heteroplasmy is necessary for the manifestation of the complete neurological phenotype in the Dutch family. This different threshold must be explained by the influence of a second factor, environmental or genetic. This factor might have a 'protective' influence in the Dutch family or alternatively a 'pathogenic' influence in the Sicilian family. A Iso for other mitochondrial mutations, additional factors are known to be involved, but have remained unidentified. ${ }^{15,16}$ Such a factor might be another mtDNA variation, as suggested for the $7445 \mathrm{~A} \rightarrow \mathrm{G}$ mutation. However, sequencing of the complete mitochondrial genome would be needed to investigate this possibility in our family.

O $n$ the other hand, nuclear genes may also influence the penetrance and expression of mitochondrial mutations. The recent finding that mice with a mutation in the myosin VIIA gene are protected from aminoglycoside ototoxicity ${ }^{17}$ suggests that the myosin VIIA gene may be a nuclear factor influencing mitochondrial mutations that predispose to aminoglycoside induced deafness.

We investigated possible statistical correlations between hearing impairment and heteroplasmy or between age and heteroplasmy. Both were not significant. The absence of a correlation between hearing thresholds and heteroplasmy is remarkable, as the causative role of the mutation is beyond doubt. $\mathrm{H}$ owever, heteroplasmy was determined in blood, and a significant correlation might be found in other tissues. $\mathrm{Up}$ to now, heteroplasmy levels in muscle have only been determined in a single patient of the D utch family and in two patients of the Sicilian family. A Ithough identical values for blood and muscle were obtained in these three cases, more patients need to be analysed to produce statistics.

It is noteworthy that three D utch family members of the maternal line reported acute hearing loss after the use of streptomycin. This suggests the involvement of the 7472insC mutation in susceptibility to ototoxic medication. However, the 7472insC mutation was not found in a set of 45 additional patients with aminoglycoside-induced hearing loss without the $1555 \mathrm{~A} \rightarrow \mathrm{G}$ mutation. This could indicate that the presence of three patients with aminoglycoside-induced deafness in the $\mathrm{D}$ utch family is purely coincidental. A Iternatively, if the 7472insC mutation does influence the sensitivity to aminoglycosides, it is probably a rare cause of aminoglycoside-induced hearing loss.

In this study we report a family in which the 7472insC mutation causes non-syndromic hearing loss in most patients, and syndromic hearing loss in a single patient. It is therefore obvious that, in addition to the $1555 \mathrm{~A} \rightarrow \mathrm{G}$ and the $7445 \mathrm{~A} \rightarrow \mathrm{G}$ mutations, the 7472insC mutation is an important mutation to be analysed in families with maternally-inherited non-syndromic hearing loss.

\section{Acknowledgements}

This study was supported by a grant from the $U$ niversity of A ntwerp to PJW, PVdH and GVC and a grant from the 'Fonds voor Wetenschappelijk Onderzoek' (FWO) to GVC. $\mathrm{KV}$ holds a pre-doctoral research position with the 'V laams instituut voor de bevordering van het wetenschappelijktechnologisch onderzoek in de industrie' (IWT), and GVC holds a research position with the FWO.DFJ and NF-G were supported by NIH/NIDCD grants R01DC01402 and R 01D C 02273, and MZ by a grant from the Telethon foundation no. 767.

\section{References}

1 Wallace DC: Diseases of the mitochondrial DNA. Annu Rev Biochem 1992; 61: 1175-1212.

2 Wallace DC: Mitochondrial diseases; genotype versus phenotype. Trends $G$ enet 1993; 9: 123-128.

3 Prezant TR, A gapian JV, Bohlman MC et al: M itochondrial ribosomal RNA mutation associated with both antibiotic-induced and non-syndromic deafness. $\mathrm{N}$ at $\mathrm{G}$ enet 1993; 4: 289-294.

4 M atthijs G, Claes S, Longo-M benza B, Cassiman J : Nonsyndromic deafness associated with a mutation and a polymorphism in the mitochondrial $12 \mathrm{~S}$ ribosomal $\mathrm{RNA}$ gene in a large Zairean pedigree. E ur J H um G enet 1996; 4: $46-51$.

5 El-Schahawi M, Lopez de M unain A, Sarrazin A M et al: Two large Spanish pedigrees with nonsyndromic sensorineural deafness and the mtDNA mutation at nt 1555 in the 12s rR NA gene: evidence of heteroplasmy. N eurology 1997; 48: 453-456.

$6 \mathrm{U}$ sami S, A be S, Kasai $\mathrm{M}$ et al: $\mathrm{G}$ enetic and clinical features of sensorineural hearing loss associated with the 1555 mitochondrial mutation. L aryngoscope 1997; 107: 483-490. 
7 Fischel-G hodsian N, Prezant TR, Bu X, O stas S: M itochondrial ribosomal RNA gene mutation in a patient with sporadic aminoglycoside ototoxicity. A m J O tolaryngology 1993; 14: 399-403.

$8 \mathrm{H}$ utchin $\mathrm{T}, \mathrm{H}$ aworth I, H igashi $\mathrm{K}$ et al: A molecular basis for human hypersensitivity to aminoglycoside antibiotics. Nucleic A cids Res 1993; 21: 4174-4179.

9 Pandya A, Xia X, Radnaabazar J et al: Mutation in the mitochondrial $12 \mathrm{~S}$ RNA gene in two families from M ongola with matrilineal aminoglycoside ototoxicity. M ed G enet 1997; 34: 169-172.

10 Reid FM, Vernham GA, Jacobs HT: A novel mitochondrial point mutation in a maternal pedigree with sensorineural deafness. H um M utat 1994; 3: 243-247.

11 Fischel-G hodsian N, Prezant TR, Fournier P, Stewart IA, $M$ aw $M$ : Mitochondrial mutation associated with nonsyndromic deafness. Am J Otolaryngology 1995; 16: 403-408.

12 Tiranti V, Chariot $\mathrm{P}, \mathrm{C}$ arella $\mathrm{F}$ et al: $\mathrm{M}$ aternally inherited hearing loss, ataxia and myoclonus associated with a novel point mutation in mitochondrial tR NA Ser(UCN) gene. H um M ol G enet 1995; 4: 1421-1427.
13 Fischel-Ghodsian N, Prezant TR, Chaltraw $\mathrm{W}$ et al: Mitochondrial gene mutations: a common predisposing factor in aminoglycoside ototoxicity. A m J O tolaryngology 1997; 18: 173-178.

14 Torroni A, Wallace DC: M itochondrial DNA variation in human population and implications for detection of mitochondrial DNA mutations of pathological significance. J Bioenerg Biomembr 1994; 26: 261-271.

15 Guan MX, Fischel-G hodsian N, A ttardi G: B iochemical evidence for nuclear gene involvement in phenotype of non-syndromic deafness associated with mitochondrial 12S rR NA mutation. Hum M ol G enet 1996; 5: 963-971.

16 Fischel-G hodsian N, Prezant TR, Fournier $P$, Stewart IA, Maw $M$ : M itochondrial mutation associated with nonsyndromic deafness. Am J Otolaryngology 1995; 16: 403-408.

17 Richardson GP, Forge A, Kros CJ, Fleming J, B rown SD M, Steel K P: M yosin VIIA is required for aminoglycoside accumulation in cochlear hair cells. J Neurosci 1997; 17: $9506-9519$. 